\title{
Recherche en médecine de premier recours?
}

La médecine de premier recours en tant que spécialité existe officiellement en Suisse depuis 25 ans - date de la création de la Société Suisse de Médecine générale (SSMG) - et la définition du médecin généraliste (ou médecin de famille) a trouvé un consensus européen en 2002. L'enseignement en médecine générale a été introduit en Suisse en 1980 par une décision de notre parlement, mais où en est le deuxième pilier d'une branche qui se veut académique: la recherche?

Ni la création en 1981 d'un groupe de travail pour la recherche de la SSMG, doublé par celui du Collège de Médecine de Premier Recours ni les vœux pieux exprimés après un séminaire sur la recherche clinique organisé par l'Académie Suisse des Sciences Médicales (ASSM) il y a 10 ans [1] n'ont réussi à faire démarrer un vrai mouvement de recherche dans ce domaine. Les raisons sont multiples: l'absence de représentations académiques véritables et reconnues par tout le monde au niveau helvétique (malgré des développements prometteurs à Berne [FIAM] et à Lausanne et Genève [création de Départements de Médecine communautaire]); le fait que trois sociétés se partagent (et quelques fois se disputent) le domaine de la médecine de premier recours (malgré les efforts louables de réunir les forces au niveau du Collège); la difficulté de définir les bases scientifiques de la médecine de premier recours; le manque de cadres formés; et encore beaucoup d'autres.

Tout ceci n'est cependant pas une raison de prononcer des oraisons funèbres [2] sur la recherche en médecine générale! Un séminaire organisé par l'ASSM à Bâle le 15 novembre 2002 qui a réuni des personnes intéressées dans ce domaine - représentants des trois sociétés impliquées en médecine de premier recours, du Collège, des facultés et de l'ASSM - a prouvé bien le contraire: la volonté existe bel et bien pour faire avancer le domaine de la recherche en médecine de premier recours. Des interventions par des invités étrangers (Prof. A. Knottnerus de Maastricht en Hollande et Prof. F. Olesen d'Aarhus au Danemark) ont montré que d'autres pays de notre taille y ont bien réussi et des groupes de travail formés ad hoc ont montré que les idées ne manquent pas, et dans quelle direction il faudra agir:

- sensibilisation des jeunes étudiants à la recherche en médecine de premier recours (summer school?);

- organisation au niveau suisse d'une maîtrise (master) en recherche en médecine de premier recours (à l'image du master en santé publique);

- formation en recherche de médecins installés prêts à participer à la recherche (à l'image des groupes «Sentinella»);

- organisations de réseaux type «Sentinella» ad hoc pour des projets bien définis;

- sensibilisations des politiciens, des responsables des caisses et des donateurs pour ce champ prometteur.

A la fin de la réunion du 15 novembre qui sera résumée dans un prochain numéro de PrimaryCare [3] tout le monde est tombé d'accord: cette fois il faut profiter de l'enthousiasme créé par les conférences et les discussions et agir afin de ne pas laisser passer l'occasion unique du consensus parmi tous les participants: la médecine de premier recours est bel et bien une spécialité académique qui mérite une recherche de pointe!

Prof. Hans Stalder, Genève Président de la commission «Recherche et réalisation en médecine appliquée» de l'ASSM

1 Stalder H. La recherche en médecine ambulatoire. Bull Méd Suisse 1993;74:1124-6.

2 Kuenzi B. 20 Jahre AG Forschung der SGAM eine festliche Trauerrede. www.ssmg.ch.

3 Kissling B. Encouragement de la recherche en médecine ambulatoire. PrimaryCare 2003;3(6): in print. www.primary-care.ch. 